\title{
Probability as certainty: Dichotomous thinking and the misuse of $p$ values
}

\author{
RINK HOEKSTRA \\ University of Groningen, Groningen, The Netherlands \\ SUE FINCH \\ University of Melbourne, Melbourne, Queensland, Australia \\ and \\ HENK A. L. KIERS and ADDIE JOHNSON \\ University of Groningen, Groningen, The Netherlands
}

\begin{abstract}
Significance testing is widely used and often criticized. The Task Force on Statistical Inference of the American Psychological Association (TFSI, APA; Wilkinson \& TFSI, 1999) addressed the use of significance testing and made recommendations that were incorporated in the fifth edition of the APA Publication Manual (APA, 2001). They emphasized the interpretation of significance testing and the importance of reporting confidence intervals and effect sizes. We examined whether 286 Psychonomic Bulletin \& Review articles submitted before and after the publication of the TFSI recommendations by APA complied with these recommendations. Interpretation errors when using significance testing were still made frequently, and the new prescriptions were not yet followed on a large scale. Changing the practice of reporting statistics seems doomed to be a slow process.
\end{abstract}

The statistical technique used most often in the behavioral sciences is also one of the most controversial: the so-called null-hypothesis significance test (hereafter called significance testing). Although significance testing has its defenders (e.g., Mulaik, Raju, \& Harshman, 1997), it has for decades been heavily criticized (e.g., Bakan, 1966; Batanero, 2000; Cohen, 1994; Cortina \& Dunlap, 1997; Falk \& Greenbaum, 1995; Harlow, Mulaik, \& Steiger, 1997; Lecoutre, Poitevineau, \& Lecoutre, 2003; Rozeboom, 1960; Schmidt, 1996; Vacha-Haase, 2001). Problems associated with significance testing include an increased risk of the so-called publication bias (e.g., Bakan, 1966); failing to take into account that there are a priori reasons for believing that the commonly tested null hypothesis of "no effect" is false (Bakan, 1966); and the crucial problem that the significance test "does not tell us what we want to know" (Cohen, 1994, p. 997).

In this study, we focus on yet another problem-namely, that significance testing encourages a binary way of interpreting results (Rosnow \& Rosenthal, 1989) and even suggests the absence of uncertainty with regard to statistical inference. Many researchers seem to rely on the outcome of the significance test as a binary indicator of importance or even effect size, and few are capable of interpreting the

Correspondence relating to this article may be sent to $\mathrm{R}$. Hoekstra, Department of Psychology, Faculty of Behavioral and Social Sciences, University of Groningen, Grote Kruistraat $2 / 1$, Groningen 9712 TS, The Netherlands (e-mail: r.hoekstra@rug.nl). $p$ value correctly (Oakes, 1986). Researchers might thus erroneously interpret the significance of an outcome as indicating the importance of an effect. However, as we all once learned, the statistical significance of an effect (i.e., rejecting the null hypothesis) does not mean that the effect is important. Moreover, lack of statistical significance (i.e., failing to reject the null hypothesis) means only that no clear evidence for rejecting the null hypothesis is available, and does not imply that the null hypothesis represents the true state of the world. For these reasons, it has been argued that binary interpretations can compromise the correct interpretation of a given study in particular (Rosnow \& Rosenthal, 1989) and, according to some, even the progress of science in general (Schmidt, 1996).

Because significance testing implies categorization of experimental outcomes, situations might arise in which it is difficult to resolve controversies about whether a particular effect exists or not. Rossi (1997) illustrated this with an example of a meta-analysis conducted on the phenomenon of spontaneous recovery of verbal learning. The meta-analysis of 39 studies included 47 experiments, of which 20 reported significant results and 27 did not. Based on the number of significant outcomes only, it is hard to judge whether spontaneous recovery exists or not. However, the meta-analysis-including measures of effect size-showed a relatively large overall effect.

When only the outcomes of significance tests or relative $p$ values (i.e., whether a $p$ value meets an arbitrary cutoff, such as $p<.05$ ) are reported, the ratio scale of the exact $p$ value is reduced to only two categories, resulting 
in the loss of information. Moreover, giving only a relative $p$ value does not give information about the degree of uncertainty.

The illusion of certainty created by the use of significance testing (Gigerenzer, 2004) may be overcome by adding measures of uncertainty to the analysis of experimental outcomes. Specifically, confidence intervals (hereafter referred to as $C I s$ ) explicitly convey the uncertainty of an estimate.

It is an open question whether the ongoing debate about the value of significance testing has led to changes in scientific practice (Finch, Cumming, \& Thomason, 2001). This debate did, however, lead to the formation of the Task Force on Statistical Inference within the American Psychological Association (TFSI, APA; Wilkinson \& TFSI, 1999). The goals of the TFSI were to elucidate some of the controversial issues surrounding application of statistics in psychology, including significance testing and its alternatives. The TFSI's recommendations were partly incorporated in the fifth edition of the APA Publication Manual (APA, 2001; Finch, Thomason, \& Cumming, 2002). The TFSI suggestions explicitly state that dichotomous accept-reject decisions are undesirable and that interval estimates, such as CIs, and effect sizes should be given for every principal outcome. As far as CIs are concerned, the guidelines state that "because confidence intervals combine information on location and precision and can often be directly used to infer significance levels, they are, in general, the best reporting strategy," and they are "therefore strongly recommended" (APA, 2001, p. 22).

Nevertheless, most researchers think of significance testing as the method for presenting their results. For example, in a study of 150 articles from the Journal of Applied Psychology, Finch et al. (2001) found that $97 \%$ of the articles contained at least one significance test. According to Masson and Loftus (2003), practicing scientists rely almost reflexively on the logic and methods associated with significance testing. Despite all the criticism, and despite the recommendations of the TFSI, significance testing seems to remain "the cornerstone of statistical analysis in social science research" (Tryon, 2001, p. 371).

Although the focus of the significance testing debate has centered on the theoretical arguments for or against it, some research has focused on the interpretation of significance testing outcomes in practice (e.g., Lecoutre et al., 2003; Oakes, 1986; Rosenthal \& Gaito, 1963; Weisburd, Lum, \& Yang, 2003). For example, Finch et al. (2001) focused on misconceptions about significance testing as revealed in published reports. In particular, they examined the way in which $p$ values were reported and the differences between how statistically significant and nonsignificant outcomes were reported with respect to the guidelines suggested by the TFSI (Wilkinson \& TFSI, 1999). Very little influence of the suggestions of the TFSI was found, presumably because too little time had passed since the publication of the report.

Studies like that of Finch et al. (2001) make clear that misuse and misinterpretation of significance testing have been problems in practice, and not just in theory. Our purpose was to examine whether the problem has diminished since the publication of the recommendations of the TFSI (Wilkinson \& TFSI, 1999) in the APA manual.

We reviewed articles submitted both before and after publication of the fifth edition of the APA Publication Manual to determine whether (1) statistical significance was equated with certainty; (2) statistically nonsignificant effects were reported as nonexistent or negligible; (3) $p$ values were reported in the manner prescribed by the APA; (4) CIs were reported; and (5) effect sizes were reported.

\section{METHOD}

\section{Materials}

All 266 "brief reports" from Psychonomic Bulletin \& Review (hereafter referred to as $P B \& R)$ Volumes 9, 10, and $11(2002,2003$, and 2004) were included. In 259 of these $4-6$ page articles, significance testing was one of the main techniques used to analyze the data. The 7 articles without a significance test were excluded from our analyses. The years 2002, 2003, and 2004 were chosen such that submission dates overlapped with the publication of the fifth edition of the APA Publication Manual in June 2001. In all, 101 of the analyzed articles were submitted before the publication of the APA manual, and 158 were submitted after its publication. We also examined 20 articles from $P B \& R$ in 1994 and 1995 to provide an earlier baseline. $P B \& R$ was chosen for its breadth and its relatively high impact factor (1.9 in 2004). Although all of the studies we examined came from $P B \& R$, the journal's quality and breadth is such that both the style and content of articles published there are likely to be representative of articles by researchers in a wide range of subdisciplines of psychology, and thus, the articles studied here can be considered a sample from the entire population of psychology articles. Of the recent articles, 93\% (242) reported both statistically significant and statistically nonsignificant test results; 17 articles reported statistically significant test results only. These findings are consistent with our view that significance testing is still prevalent in psychonomic science.

Given that it is likely that more interpretation errors are made in less prominent journals than in prominent journals such as $P B \& R$, the CIs in the Results and Discussion section can be considered a conservative estimate of the proportion of interpretation errors in those less prominent journals.

\section{Checklist}

A 13-item checklist was developed for recording ways of reporting significance testing. Items fell into five categories, described below. Items were scored " 1 " if at least one occurrence was found in the article, and " 0 " otherwise.

Reporting statistical significance as certainty. Concluding that a statistically significant test result implies with certainty that the effect is present in the population is incorrect because such a conclusion ignores the possibility of a Type I error. Statements such as "we demonstrated that A influences B"; "we showed that A is larger than B"; and "given this result, we know that there is an effect" were considered errors of this type, whereas statements such as "this suggests that there is an effect" and "there probably is an effect" were not considered incorrect. Very general phrases such as "Group A scored higher than Group B" are ambiguous and could arguably be interpreted as referring to the sample. Thus, they were not counted as reporting statistical significance as certainty, whereas phrases such as "men score higher than women" were.

Reporting that there is no effect or a negligibly small effect. It is a serious mistake to interpret a statistically nonsignificant effect as proof of the absence of an effect in the population. Phrases such as "there is no effect," "there was no evidence for" (combined with an effect in the expected direction), "the nonexistence of the effect," "no effect was found," "are equally affected," "there was no main effect," "A and $B$ did not differ," or "the significance test reveals that there is no 
difference" were taken as evidence of this mistake. It is also incorrect to consider an effect negligibly small when this is based only on the outcome of a significance test. Therefore, we also coded whether a statistically nonsignificant effect was described as a negligibly small effect. We considered this the case when statements like "there is hardly an effect," "the effect is negligible," or "the almost absent effect" were used without relating the result to a $\mathrm{CI}$, a standardized effect size, or an unstandardized effect size with a measure of variability.

Reporting exact versus relative $p$ values. According to the APA Publication Manual, exact $p$ values should always be reported, except when appearing in large tables of correlations or complex tables of path coefficients. We coded use of relative $p$ values (defined as $p$ smaller or larger than a fixed value; e.g., $p<.05$ or $p>.10$ ) and of exact $p$ values for both statistically significant and statistically nonsignificant results. We also checked whether effects were reported as statistically significant or nonsignificant without mentioning a $p$ value. We did not require that the term statistically be used when we recorded this item.

Reporting confidence intervals. Reporting a point estimate or significance test result without presenting a CI runs counter to APA guidelines. We coded the use of CIs, including visual representations and numerical reports of an interval with an associated confidence level. We also coded whether some other visually represented measure of error was provided, such as a standard error or standard deviation.

Reporting effect size. Reporting $p$ values alone does not make it possible to draw conclusions about the magnitude of an effect within a population. For that purpose, an effect size based on the sample should be reported. We used broad as well as narrow criteria to check for the mentioning of effect sizes. The broad criteria included standardized and unstandardized effect sizes, including sample means. The narrow criteria included only standardized effect sizes.

\section{Procedure}

All 259 articles were coded by the first author; 16 articles (6\%) were cross-coded by the second author. In total, 192 binary items were cross-coded. For $98 \%$ of those items, the coding was identical. Although the items differed in their complexity as far as scoring was concerned, the proportion of agreement indicates that there was little disagreement between the authors. The four instances of disagreement were on the "accepting the null hypothesis" item, in which the first author apparently required stronger evidence. To be more conservative, we used his codes.

\section{Analysis}

Table 1 shows the results for all checked items. For illustration, we provide the percentage of articles in 1994-1995 using each reporting practice. In the third and fourth columns, the percentage of articles published in 2002-2004 using each practice is given, along with a $95 \% \mathrm{CI}$. The second, fifth, and sixth columns represent descriptive statistics only, and therefore the $95 \%$ CIs are not given. All the CIs for percentages were calculated using the Wilson estimate procedure (see Moore \& McCabe, 2003).

We also investigated the change in reporting practices from before and after the publication of the fifth edition of the APA Publication Manual. Columns 5 and 6 of Table 1 give the percentages of articles using each practice before and after June 2001. To make inferences about the change in reporting practice, we used linear logistic regression to predict occurrence of a given reporting practice from submission time (measured in years relative to June 2001). The results of a logistic regression are best interpreted in terms of the odds of an event occurring - that is, the probability of the event occurring over the probability of it not doing so. Specifically, we used linear logistic regression to estimate the relation between the submission time and the odds of the specific reporting practices being used. For simplicity, we report the weight for the odds - that is, the factor by which the odds changed each year. For example, if the weight for the odds of reporting significance as certainty were 0.5 , this would mean that the odds halved from one year to the next. If the weight were 1 , it would mean that the odds did not change from year to year. If the weight were 2 , the odds would have doubled over a year. We refer to the weight for the odds estimated by linear logistic regression as the estimated annual change in odds factor. The percentages of articles using a practice before and after the fifth edition of the APA manual (Columns 5 and 6 in Table 1) also can be used for the interpretation of the odds.

\section{RESULTS AND DISCUSSION}

\section{Reporting Significance as Certainty}

In total, $19 \%$ (CI: $14 \%, 24 \%$ ) of the articles contained at least one phrase in which authors indicated their certainty of an effect in the population. The proportion of such statements dropped from $23 \%$ before to $16 \%$ after

Table 1

Percentages of Articles (With 95\% Confidence Intervals) Using 13 Reporting Practices, for Articles Published in 1994-1995 (20 Articles) and 2002-2004 (259 Articles), and Annual Change in Odds Factor for Articles Published Before (101) and After (158) Publication of the 5th Edition of the APA Manual

\begin{tabular}{|c|c|c|c|c|c|c|c|}
\hline \multirow[b]{2}{*}{ Reporting Practice } & \multirow[b]{2}{*}{ 1994-1995 } & \multicolumn{2}{|c|}{ 2002-2004 } & \multirow{2}{*}{$\begin{array}{l}\text { Before } \\
\text { 5th } \\
\text { Edition }\end{array}$} & \multirow{2}{*}{$\begin{array}{l}\text { After } \\
\text { 5th } \\
\text { Edition }\end{array}$} & \multicolumn{2}{|c|}{$\begin{array}{l}\text { Annual Change in } \\
\text { Odds Factor }\end{array}$} \\
\hline & & $\%$ & $95 \% \mathrm{CI}$ & & & Weight & $95 \% \mathrm{CI}$ \\
\hline Significance as certainty & 10 & 19 & 14,24 & 23 & 16 & 0.83 & $0.62,1.09$ \\
\hline No effect* ${ }^{*}$ & 41 & 60 & 53,66 & 60 & 60 & 1.00 & $0.88,1.25$ \\
\hline No or negligible effect* & 41 & 63 & 57,69 & 67 & 61 & 0.88 & $0.70,1.11$ \\
\hline \multicolumn{8}{|l|}{ Statistically significant results } \\
\hline with exact $p$ values & 25 & 21 & 17,27 & 24 & 20 & 1.02 & $0.78,1.32$ \\
\hline with relative $p$ values & 80 & 84 & 79,88 & 86 & 83 & 0.88 & $0.65,1.18$ \\
\hline with no $p$ value & 25 & 20 & 15,25 & 19 & 20 & 1.14 & $0.86,1.49$ \\
\hline \multicolumn{8}{|c|}{ Statistically nonsignificant results ${ }^{*}$} \\
\hline with exact $p$ values & 24 & 45 & 39,52 & 46 & 45 & 0.94 & $0.75,1.17$ \\
\hline with relative $p$ values & 41 & 32 & 26,38 & 39 & 27 & 0.83 & $0.66,1.06$ \\
\hline with no $p$ value & 82 & 68 & 62,74 & 61 & 73 & 1.09 & $0.86,1.37$ \\
\hline Confidence intervals & 0 & 5 & 3,9 & 5 & 6 & $*$ & \\
\hline Error bars & 15 & 37 & 31,43 & 31 & 41 & 1.17 & $0.93,1.46$ \\
\hline Measure of effect size & 100 & 99 & 97,100 & 100 & 99 & ** & \\
\hline Standardized effect size & 0 & 1 & 0,4 & 0 & 2 & $*$ & \\
\hline
\end{tabular}

"Our analyses excluded articles without statistically nonsignificant results (3 articles in 1994-1995, 17 in 2002-2004). "*dds factors were not calculated for proportions close to 0 or 1 , because in that case there are hardly any observations for one of the two categories of the binary variable. 
publication of the fifth edition of the APA Publication Manual. The estimated annual change in odds factor was 0.83 . This trend is in a "progressive" direction, but note that for the interpretation of the estimated change in odds factors, CIs should be taken into account (see Table 1), and it can be seen that these are generally rather wide.

\section{Reporting That There Is No Effect or That an Effect Is Negligibly Small}

We found the serious mistake of accepting the null hypothesis and claiming no effect in $60 \%$ (CI: $53 \%, 66 \%$ ) of the articles that reported statistically nonsignificant results. This percentage was found both before and after the publication of the APA manual.

There was a claim of no effect or of a negligible effect without sufficient statistical evidence for the negligibility of the effect in $63 \%$ (CI: $57 \%, 69 \%$ ) of the articles. This percentage dropped from $67 \%$ (before the fifth edition) to $61 \%$ (after the fifth edition), giving an estimated annual change in odds factor of 0.88 .

\section{Reporting Exact Versus Relative $p$ Values}

For this analysis, we excluded articles that contained only relative $p$ values in tables. Such reporting is consistent with APA recommendations, which state that when reporting exact $p$ values can be awkward, such as in large tables, relative $p$ values may be reported instead. Overall, $52 \%$ of the articles contained exact $p$ values, with only a $1 \%(\mathrm{CI}$ : $-11 \%, 13 \%)$ difference between articles submitted before and after June 2001. The estimated annual change in odds factor was 1.20 , indicating a possible increase after June 2001. Use of relative $p$ values was also relatively stable, but it diminished slightly, by $4 \%$ (CI: $-13 \%, 0 \%)$, for submissions before $(88 \%)$ and after $(84 \%)$ the most recent APA manual.

We also checked whether the reporting of exact and relative $p$ values and the reporting of a result without a $p$ value differed for statistically significant and statistically nonsignificant results. Articles in which no statistically nonsignificant results were reported were excluded from the latter analysis.

Clear differences were found between the ways in which statistically significant and nonsignificant results were reported, as can be seen in Table 1. Exact or no $p$ values were given more often for statistically nonsignificant results than for statistically significant results, whereas the reverse held for relative $p$ values.

\section{Reporting Uncertainty}

Only $5 \%$ of articles before the publication of the fifth APA Publication Manual reported CIs, and $6 \%$ of articles after. Overall, we found other measures of uncertainty in $37 \%$ (CI: $31 \%, 43 \%$ ) of the articles, rising from $31 \%$ of pre-June 2001 to $41 \%$ of post-June 2001 submissions (CI for the difference: $0 \%, 21 \%$ ). The estimated annual change in odds factor was 1.17 .

Table 1 shows clearly that the reporting of CIs and measures of uncertainty in general is still limited. Although an increasing trend is visible for all measures, it can be safely concluded that CIs, as well as measures of uncertainty in general, are still not widely reported.

\section{Reporting Effect Size}

An indication of standardized or unstandardized (such as giving means) effect size was found in all but two articles $(99 \%$; CI: $97 \%, 100 \%)$. Some measure of effect size was given almost universally. However, a standardized effect size was given in only three articles. This finding indicates that although effect size in the broad sense was reported almost universally, standardized effect size was almost never reported.

\section{Long-Term Trends}

The scoring of 20 articles from $P B \& R$ in 1994-1995 showed results comparable to those obtained from the pre-June 2001 articles. Although differences can of course be seen between the 1994-1995 and the later articles in Table 1, these may be due to the relatively small sample size used for the 1994 and 1995 articles. It seems safe to conclude that there have not been radical changes in reporting practices in the past decade. This finding supports our general view that if there have been any long-term changes in the first place, those changes have not been large.

\section{GENERAL DISCUSSION}

Our review of $266 P B \& R$ articles published from 2002 to 2004 made clear that significance testing is still overwhelmingly used in psychological research, with $97 \%$ of the articles containing at least one significance test. Cases of misinterpretation and insufficient information abounded in the studied articles, with more than half of the articles claiming a null effect. Moreover, the APA guidelines published in 2001 have not been followed consistently: Although some measure of effect size was found in a large majority of the articles, exact $p$ values were not consistently reported, and CIs were found in only a small minority of the articles.

At least one claim of certainty or importance on the basis of a statistically significant outcome was found in $19 \%$ of the articles. We suspect that this number underestimates the proportion of researchers who view statistical significance as indicating importance or who interpret a statistically significant effect as a proven population effect. For instance, frequently used statements such as "the significance test showed that the placebo group scored higher than the treatment group" at least suggest that the conclusion also holds in the population. Such ambiguous cases were not counted, so our estimate of reporting significance as certainty or importance is probably an underestimation. Both claiming certainty on the basis of a significant finding (Rosnow \& Rosenthal, 1989) and accepting the null hypothesis suggest binary thinking on the part of a researcher. Our results, especially those for statistically nonsignificant findings, are consistent with the idea that significance testing encourages binary thinking.

Despite the fact that APA guidelines prescribe the reporting of exact $p$ values, we found many instances of 
relative $p$ values or of no $p$ values at all. The pattern of reporting $p$ values (exact, relative, or not at all) varied markedly according to whether statistical significance was achieved. Such a pattern is consistent with our view that significance testing is accompanied by binary thinking. "Successes" (rejecting the null hypothesis) are reported relative to the standard for success (rather than more precisely), and $p$ values associated with "failures" (failure to reject the null hypothesis) are not deemed interesting enough to be reported.

Although highly recommended by the APA, CIs are far from being generally used as a standard way of presenting results. We did see an increase in the use of measures of uncertainty in general, but the frequencies were too small to warrant general conclusions. An explanation for the infrequent use of CIs could be that changes in the ways researchers report data are usually slow, so it may be premature to state that the APA Publication Manual will have no influence as far as the frequency of the use of CIs is concerned.

In the introduction, we stated that binary thinking could impede the progress of science in general, that significance testing can be regarded as encouraging binary thinking, and that following APA guidelines should lead to a decrease in binary thinking. Our results show that the new guidelines are not yet followed on a large scale, although some small trends toward improvement can be discerned. Diminishing the occurrence of binary thinking in science-which we think essential-will likely be a difficult and time-consuming process.

\section{REFERENCES}

American Psychological Association (2001). Publication manual of the American Psychological Association (5th ed.). Washington, DC: Author.

BAKAN, D. (1966). The test of significance in psychological research. Psychological Bulletin, 66, 423-437.

Batanero, C. (2000). Controversies around the role of statistical tests in experimental research. Mathematical Thinking \& Learning, 2, 75-97.

CoHEN, J. (1994). The earth is round $(p<.05)$. American Psychologist, 49, 997-1003.

CoRTINA, J. M., \& DUnLAP, W. P. (1997). On the logic and purpose of significance testing. Psychological Methods, 2, 161-172.

FalK, R., \& Greenbaum, C. W. (1995). Significance tests die hard: The amazing persistence of a probabilistic misconception. Theory \& Psychology, 5, 75-98.

Finch, S., Cumming, G., \& Thomason, N. (2001). Reporting of statisti- cal inference in the Journal of Applied Psychology: Little evidence of reform. Educational \& Psychological Measurement, 61, 181-210.

Finch, S., Thomason, N., \& Cumming, G. (2002). Past and future American Psychological Association guidelines for statistical practice. Theory \& Psychology, 12, 825-853.

GIGERENZER, G. (2004). Mindless statistics. Journal of Socio-Economics, $33,587-606$.

HaRlow, L. L., Mulaik, S. A., \& Steiger, J. H. (1997). What if there were no significance tests? Mahwah, NJ: Erlbaum.

Lecoutre, M.-P., Poitevineau, J., \& Lecoutre, B. (2003). Even statisticians are not immune to misinterpretations of null hypothesis tests. International Journal of Psychology, 38, 37-45.

Masson, M. E. J., \& LoFTUs, G. R. (2003). Using confidence intervals for graphically based data interpretation. Canadian Journal of Experimental Psychology, 57, 203.

MOORE, D. S., \& MCCABE, G. P. (2003). Introduction to the practice of statistics. New York: Freeman.

Mulaik, S. A., Raju, N. S., \& Harshman, R. (1997). There is a time and place for significance testing. In L. L. Harlow, S. A. Mulaik, \& J. H. Steiger (Eds.), What if there were no significance tests? (pp. 65116). Mahwah, NJ: Erlbaum.

OAKES, M. (1986). Statistical inference: A commentary for the social and behavioural sciences. Chichester, U.K.: Wiley.

Rosenthal, R., \& GaIto, J. (1963). The interpretation of levels of significance by psychological researchers. Journal of Psychology: Interdisciplinary \& Applied, 55, 33-38.

RosNow, R. L., \& Rosenthal, R. (1989). Statistical procedures and the justification of knowledge in psychological science. American Psychologist, 44, 1276-1284.

Rossi, J. S. (1997). A case study in the failure of psychology as a cumulative science: The spontaneous recovery of verbal learning. In L. L. Harlow, S. A. Mulaik, \& J. H. Steiger (Eds.), What if there were no significance tests? (pp. 175-197). Mahwah, NJ: Erlbaum.

RozeвоOM, W. W. (1960). The fallacy of the null-hypothesis significance test. Psychological Bulletin, 57, 416-428.

SCHMIDT, F. L. (1996). Statistical significance testing and cumulative knowledge in psychology: Implications for training of researchers. Psychological Methods, 1, 115-129.

TRYON, W. W. (2001). Evaluating statistical difference, equivalence, and indeterminancy using inferential confidence intervals: An integrated alternative method of conducting null hypothesis statistical tests. Psychological Methods, 6, 371-386.

VACHA-HAASE, T. (2001). Statistical significance should not be considered one of life's guarantees: Effect sizes are needed. Educational \& Psychological Measurement, 61, 219-224.

WeISBURD, D., LUM, C. M., \& YANG, S.-M. (2003). When can we conclude that treatments or programs "don't work"? Annals of the American Academy of Political \& Social Science, 587, 31-48.

WilKinson, L., \& THE APA TASK ForCe ON STATISTICAL InfERENCE (1999). Statistical methods in psychology journals: Guidelines and explanations. American Psychologist, 54, 594-604.

(Manuscript received July 28, 2005 revision accepted for publication April 25, 2006.) 